\title{
Synthesis and characterization of poly(propylene imine)-dendrimer-grafted gold nanoparticles as nanocarriers of doxorubicin
}

Marzieh Golshan, Mehdi Salami-Kalajahi ${ }^{*}$, Mina Mirshekarpour, Hossein Roghani-Mamaqani ${ }^{*}$, Maryam Mohammadi

Department of Polymer Engineering, Sahand University of Technology, P.O. Box 51335-1996, Tabriz, Iran

Institute of Polymeric Materials, Sahand University of Technology, P.O. Box 51335-1996, Tabriz, Iran

* Correspondence concerning this article should be addressed to

Mehdi Salami-Kalajahi at m.salami@sut.ac.ir, Tel. +98 413345 9097, Fax: +98 4133444313

Hossein Roghani-Mamaqani at r.mamaghani@sut.ac.ir, Tel. +98 413345 9104, Fax: +98 41

33444313 


\begin{abstract}
The aim of current work is synthesis $4^{\text {th }}$-generation-poly(propylene imine) (PPI)-dendrimer modified gold nanoparticles (Au-G4A) as nanocarriers for doxorubicin (DOX) and studying in vitro drug release kinetics from nanocarriers into different media. Accordingly, AuNPs were synthesized by reduction of chloroauric acid $\left(\mathrm{HAuCl}_{4}\right)$ aqueous solution with trisodium citrate and modified with cysteamine to obtain amine-functionalized $\left(\mathrm{Au}-\mathrm{NH}_{2}\right)$ nanoparticles. $\mathrm{Au}-\mathrm{NH}_{2}$ nanoparticles were used as multifunctional cores and participated in Michael addition of acrylonitrile and reduction process by lithium aluminum hydride (LAH) to synthesize Au-G4A nanoparticles. Also, peripheral primary amine groups of Au-G4A were conjugated with folic acid (FA) (Au-G4F) to study the bioconjugation effect on drug release behavior of nanostructures. Ultraviolet spectroscopy (UV-visible), atomic force microscopy (AFM), transmission electron microscopy (TEM), Fourier transform infrared spectroscopy (FT-IR), and thermal gravimetric analysis (TGA) were used to approve the synthesis of different nanostructures. Finally, Au-G4A and Au-G4F samples were loaded with DOX and exposed to environments with different $\mathrm{pH}$ values to examine the release properties of nanostructures. Also, drug release kinetics was investigated by fitting of experimental data with different release models. As a result, synthesized dendritic structures showed Higuchi and Korsmeyer-Peppas models release behavior due to better solubility of drug in release media with respect to dendrimer cavities and drug release through polymeric matrix respectively.
\end{abstract}

Keywords: Gold nanoparticles; Poly(propylene imine) dendrimer; Doxorubicin; Drug release kinetics 


\section{Introduction}

Gold nanoparticles (AuNPs) as non-toxic and biologically compatible nanoparticles have gotten increasing attention in different fields such as biomedicals [1], hybrid nanomaterials [2], etc. Also, Surface Plasmon resonance (SPR) as a unique optical property is conferred by the interaction of light with electrons on the surface of gold nanoparticles [3]. These have resulted in application of AuNPs in different drug delivery systems [4-5]. Beside this, dendrimers as three-dimensional, highly-branched and monodispersed polymeric nanostructures have been used in drug delivery systems [6-7]. They are synthesized via an iterative sequence of reactions such as Michael addition, alkylation and reduction [8-9]. Poly(propylene imine) (PPI) dendrimer as an amine-terminated dendrimer is synthesized via Michael addition of acrylonitrile to an amine-capped core and subsequent reduction of nitrile groups to primary amines by reduction catalysts [10]. However, due to cytotoxicity of dendrimers originated from peripheral amine groups, surface primary amine groups have been conjugated with biocompatible compounds such as folic acid (FA) [11] and polymeric chains [12]. In this field, FA is one of the most promising candidates with the potential for cancer-cell specific targeting due to its high affinity for folate receptors (FRs) [13]. However, conjugation of FA with PPI dendrimers may affect the drug release behavior of dendrimers.

Nowadays, different works have been reported about PPI dendrimer/gold hybrid nanoparticles [1416]. In some cases, they have been utilized as catalyst for reduction of phenol derivatives [14] whereas in other cases they have been used in cancer therapy [15-16]. However, no work has reported the generation growth from the surface of AuNPs as well as drug release kinetics from these hybrid nanoparticles even in FA-conjugated or -nonconjugated forms. On the other hand, PPI dendrimers can also be used to modify AuNPs to enhance their therapeutic properties via 
improvement of the cytocompatibility and cellular uptake [16]. Furthermore, attaching dendrimer onto the surface of nanoparticles results in easy purification process [17].

In this work, AuNPs have been prepared by reduction of $\mathrm{HAuCl}_{4}$ with trisodium citrate and subsequent reaction with cysteamine. Then, amine-functionalized AuNPs have been used as multifunctional cores and acrylonitrile has been added to amines via Michael addition reaction to prepare half-generation-PPI-modified AuNPs. To reduce nitrile groups to primary amines, a hydrogenation process by lithium aluminum hydride (LAH) has been utilized until $4^{\text {th }}$-generation PPI dendrimer has been grafted onto surface of nanoparticles. Also, effect of FA conjugation with peripheral amine groups on drug release behavior of hybrid nanoparticles has been investigated. Finally, doxorubicin (DOX) as an anti-cancer drug was loaded onto different synthesized hybrid nanoparticles and its release behavior has been studied in different conditions. Also, release kinetics has been studied by fitting of release data with different drug release models.

\section{Experimental Section}

\subsection{Synthesis of Au nanoparticles}

Gold powder $(0.860 \mathrm{~g})$ was dissolved in $800 \mathrm{~mL}$ of 3:1 (v/v) mixture of concentrated $\mathrm{HCl}$ and $\mathrm{HNO}_{3}$ at $300{ }^{\circ} \mathrm{C}$ to obtain $\mathrm{HAuCl}_{4}$. After evaporation of solvent, $\mathrm{HAuCl}_{4}$ was diluted to $20 \mathrm{~L}$ with distilled water to achieve $0.2 \mathrm{mM}$ aqueous solution of $\mathrm{HAuCl}_{4}$.

Turkevich method was used to synthesize the colloidal AuNPs via reduction of $\mathrm{HAuCl}_{4}$ by trisodium citrate [18]. Accordingly, an aqueous solution of $\mathrm{HAuCl}_{4}(150 \mathrm{~mL}, 0.2 \mathrm{mM})$ was heated to the boiling temperature and aqueous solution of trisodium citrate $(50 \mathrm{~mL}, 1.8 \mathrm{mM})$ was added 
to reaction media. In a few seconds, bluish color appeared due to formation of gold nuclei. Then, in a few minutes, the solution turned red-wine color because of the formation of nanoparticles.

\subsection{Synthesis of cysteamine-functionalized gold nanoparticles $\left(\mathrm{Au}-\mathrm{NH}_{2}\right)$}

AuNPs were functionalized with cysteamine by adding $0.2 \mathrm{M}$ aqueous solution of cysteamine hydrochloride to colloidal AuNPs. Accordingly, $1 \mathrm{~mL}$ aqueous solution of cysteamine was added to $150 \mathrm{~mL}$ colloidal AuNPs and reaction was continued for $20 \mathrm{~min}$ at room temperature and darkness. Au- $\mathrm{NH}_{2}$ nanoparticles were separated and purified by several centrifugations at 10000 rpm for 30 min and drying in vacuum at $40{ }^{\circ} \mathrm{C}$ overnight.

\subsection{Synthesis of PPI-dendrimer-grafted AuNPs}

To graft nitrile-terminated first generation of PPI dendrimer (G1N) onto surface of AuNPs, a previously described divergent method was used with some modifications [19]. To this end, Au$\mathrm{NH}_{2}$ (1.0 g, containing $2.4 \mathrm{mmol}$ amine moieties per $1.0 \mathrm{~g}$ of Au- $\left.\mathrm{NH}_{2}\right)$ was used as an initial core and dispersed in deionized water $(10 \mathrm{~mL})$ via ultrasonication $(30 \mathrm{~min})$. ACN $(0.8 \mathrm{~mL}, 12.0 \mathrm{mmol})$ was added dropwise at room temperature and dark room condition during $1 \mathrm{~h}$ to participate in Michael addition reaction. Reaction was continued for $1 \mathrm{~h}$ and then, media was heated to $80^{\circ} \mathrm{C}$ and reaction was continued for more $1 \mathrm{~h}$ to complete the addition reaction. The obtained suspension was centrifuged (10000 rpm) and washed with water three times. Finally, the remaining ACN and solvent was removed under vacuum at $50{ }^{\circ} \mathrm{C}$ for $48 \mathrm{~h}$ to obtain $\mathrm{Au}-\mathrm{G} 1 \mathrm{~N}$ nanoparticles.

To obtain amine-terminated first generation of PPI-dendrimer-grafted AuNPs (Au-G1A), nitrile groups were reduced to primary amines via a homogeneous reduction method by LAH. 
Accordingly, Au-G1N (1.0 g, containing $2.4 \mathrm{mmol}$ nitrile moieties per $1.0 \mathrm{~g}$ of Au-G1N) was dispersed in diethyl ether $(20 \mathrm{~mL})$ via ultrasonication $(30 \mathrm{~min})$ and a mixture of LAH $(0.19 \mathrm{~g}, 5$ $\mathrm{mmol})$ in diethyl ether $(60 \mathrm{~mL})$ was added dropwise and reaction was continued for $4 \mathrm{~h}$ at $34{ }^{\circ} \mathrm{C}$. Then, reaction mixture was placed in ice-water bath and deionized water $(10 \mathrm{~mL})$ was added dropwise and stirring was continued for $1 \mathrm{~h}$. After separating diethyl ether, an aqueous solution of $\mathrm{HCl}(\mathrm{pH}=3)$ was added to aqueous media to dissolve salts produced during reaction. The reaction mixture was then filtered via $0.22 \mu \mathrm{m}$ PTFE membrane filter and dried under vacuum at $50{ }^{\circ} \mathrm{C}$ for $48 \mathrm{~h}$ to obtain Au-G1A. Scheme 1 shows the reaction route to achieve Au-G1A nanoparticles. PPIdendrimer-grafted AuNPs up to fourth generation were prepared by repetition of all the above steps consecutively, with increasing quantity of ACN and subsequently LAH. Also, reaction time of Michael addition was increased $1 \mathrm{~h}$ per each generation increase.

\section{Scheme 1: The reaction route to achieve Au-G1A nanoparticles}

\subsection{Conjugation of FA with surface of Au-G4A nanoparticles}

Firstly, Au-G4A was reacted with glycidol to achieve Au-G4OH via previously described method [20-21]. Au-G4A (0.2 g) was dispersed in methanol (10 mL) via ultrasonication (30 min). A solution of glycidol $(0.14 \mathrm{~mL})$ in methanol $(10 \mathrm{~mL})$ was added dropwise to reaction media and the mixture was stirred at $60{ }^{\circ} \mathrm{C}$ under nitrogen for $24 \mathrm{~h}$. The mixture was centrifuged and obtained powder was washed with methanol and centrifuged three more times. Finally, product was dried under vacuum at $50{ }^{\circ} \mathrm{C}$ for $48 \mathrm{~h}$.

To synthesize FA-conjugated nanoparticles (Au-G4F), a previously described method with some changes was utilized [22]. FA (0.28 g), DCC $(0.133 \mathrm{~g})$ and DMAP $(0.026 \mathrm{~g})$ were dissolved in 
DMSO (10 mL) and Au-G4OH (0.2 g) was dispersed in media via ultrasonication (30 min). Then, the mixture was stirred for $72 \mathrm{~h}$ at $50^{\circ} \mathrm{C}$ and centrifuged to obtain Au-G4F. To further purification, product was washed with water and centrifuged three more times and dried under vacuum at 50 ${ }^{\circ} \mathrm{C}$ for $48 \mathrm{~h}$. Scheme 2 shows the route that we used to achieve Au-G4F nanoparticles.

\section{Scheme 2: The route to achieve Au-G4F nanoparticles}

\subsection{Encapsulation of DOX in Au-G4A and Au-G4F dendrimers}

Aqueous solution of DOX (1 mg/mL, $6 \mathrm{~mL})$ was mixed with Au-G4A or Au-G4F dendrimer (24 $\mathrm{mg}$ ), and the solution was tuned to the desired $\mathrm{pH}$ with subsequent shaking for $48 \mathrm{~h}$ in dark conditions. The DOX-loaded nanoparticles were separated by centrifugation and the loaded drug content was obtained from the drug concentrations before and after loading by means of UVvisible absorption at $480 \mathrm{~nm}$.

\subsection{In vitro drug release studies}

The DOX-loaded Au-G4A or Au-G4F aqueous solutions $(4 \mathrm{mg} / \mathrm{mL}, 5 \mathrm{~mL})$ in cellulose dialysis bag $(\mathrm{MWCO}=12000)$ were submerged in phosphate-buffered saline $(\mathrm{PBS}, 100 \mathrm{~mL})$ stirring at $200 \mathrm{rpm}\left(37 \pm 2{ }^{\circ} \mathrm{C}\right)$ for $48 \mathrm{~h}$ at two $\mathrm{pH}$ values (5.3 and 7.4). At predetermined intervals of time, 1-mL aliquots were withdrawn and replaced with an equal volume of fresh medium. The DOX concentrations were determined by UV-visible absorption at $480 \mathrm{~nm}$ according to a pure DOX calibration curve. 


\subsection{Instrumentation}

Ultraviolet visible (UV-vis.) absorptions from liquid samples were recorded on a Perkin-Elmer Lambda 9 instrument. Samples were analyzed immediately after completion of reactions. Atomic force microscopy (AFM) images were recorded using a AFM apparatus from Ara Pajouhesh Co., Iran. The scanning probe microscope (SPM) was equipped with a silicon cantilever, which was operated in the tapping mode. A droplet of colloidal Au nanoparticles (modified or unmodified) was deposited on freshly cleaved mica slide. The droplet was then carefully washed after allowing the sample to sit for about 10 minutes. To dry the sample before scanning, it was left $2 \mathrm{~h}$ in a dust protected heater. To calculate particle size and particle size distribution, BELView 6.2 was used as free image processing software considering about 50 individual nanoparticles. A transmission electron microscope (TEM), Tescan Mira, with an accelerating voltage of $120 \mathrm{kV}$ was used to study the morphology of hybrid nanoparticles. All the samples were prepared by a drop-dry method on carbon-coated copper grids. Fourier transform infrared (FT-IR) spectroscopy was performed by means of a Bruker Tensor 27 FT-IR spectrophotometer, in the range between 500 and $4000 \mathrm{~cm}^{-1}$ with a resolution of $4 \mathrm{~cm}^{-1}$. An average of 24 scans has been carried out for each sample. The samples were prepared on a $\mathrm{KBr}$ pellet in vacuum desiccators. Thermal gravimetric analyses (TGA) were carried out by means of a PL thermo-gravimetric analyzer (Polymer Laboratories, TGA 1000, UK). All samples (about $10 \mathrm{mg}$ ) were heated from ambient temperature to $700{ }^{\circ} \mathrm{C}$ at a heating rate of $10{ }^{\circ} \mathrm{C} / \mathrm{min}$ and nitrogen as the purging gas was used at a flow rate of $50 \mathrm{~mL} / \mathrm{min}$. 


\section{Results and discussion}

\subsection{Preparation and surface modification of AuNPs}

The addition of cysteamine to colloidal AuNPs gives cysteamine-stabilized AuNPs ( $\left.\mathrm{Au}-\mathrm{NH}_{2}\right)$ due to formation of Au-S covalent bond [23]. Figure 1 shows the UV-vis. spectra of AuNPs and Au$\mathrm{NH}_{2}$ nanoparticles. The characteristic peak of AuNPs appears at $520 \mathrm{~nm}$ and indicates the formation of stable gold nanoparticles with diameter of $15.8 \mathrm{~nm}$ according to the equation 1 [24]:

$d(n m)=2.99 \lambda_{\max }-1539$

where, $d$ and $\lambda_{\max }$ are diameter of nanoparticles and wavelength of the peak respectively. After treatment with cysteamine, characteristic peak of UV-vis. shifts to $522 \mathrm{~nm}$ and broadens due to coupled Plasmon resonance and indicates attachment of the cysteamine onto AuNPs surface [25].

\section{Figure 1: UV-vis. spectra of AuNPs and $\mathrm{Au}-\mathrm{NH}_{2}$ nanoparticles}

Particles size and particle size distribution of AuNPs and $\mathrm{Au}-\mathrm{NH}_{2}$ nanoparticles are calculated using AFM images as shown in Figure 2. The number average particle size $\left(D_{n}\right)$, weight average particle size $\left(\mathrm{D}_{\mathrm{w}}\right)$, and polydispersity (PDI) are calculated according to the equations (2)-(4) [26].

$$
D_{n}=\frac{\sum_{1}^{n} n_{i} D_{i}}{\sum_{1}^{n} n_{i}}
$$


$D_{w}=\frac{\sum_{1}^{n} n_{i} D_{i}^{4}}{\sum_{1}^{n} n_{i} D_{i}^{3}}$

$P D I=\frac{D_{w}}{D_{n}}$

where, $\mathrm{n}_{\mathrm{i}}$ and $\mathrm{D}_{\mathrm{i}}$ are the number of nanoparticles and diameter respectively. According to the results of AuNPs, $D_{n}, D_{w}$, and PDI are $14.4 \mathrm{~nm}, 15.8 \mathrm{~nm}$ and 1.097 respectively. This shows that nanoparticles have narrow size distribution and results agree $\mathrm{UV}$-vis. data. Also, $\mathrm{D}_{\mathrm{n}}, \mathrm{D}_{\mathrm{w}}$, and PDI of $\mathrm{Au}-\mathrm{NH}_{2}$ nanoparticles are calculated $14.8 \mathrm{~nm}, 16.1 \mathrm{~nm}$ and 1.088 respectively. This shows that treatment of AuNPs with cysteamine does not affect the particle size and particle size distribution as proved by UV-vis. results.

Figure 2: AFM images and particle size distributions of AuNPs and $\mathrm{Au}-\mathrm{NH}_{2}$ nanoparticles

The morphological study of $\mathrm{Au}-\mathrm{NH}_{2}$ nanoparticles and PPI-dendrimer-grafted AuNPs (Au-G4A) was performed by TEM images. As shown in Figure 3, AuNPs have spherical shape and individual nanoparticles are observed. Also, Au-G4A results in a spherical-like shape and a core-shell structure originated from a slightly deep contrast of core and a light contrast of shell. This arose from the diff erent mass contrasts between the Au core and dendritic shell.

Figure 3: TEM image of $\mathrm{Au}-\mathrm{NH}_{2}$ and Au-G4A nanoparticles 
The FT-IR spectra of different modified AuNPs are depicted in Figure 4. According to the results, $\mathrm{Au}-\mathrm{NH}_{2}$ nanoparticles exhibited a characteristic peak of cysteamine at $1465 \mathrm{~cm}^{-1}$ originating from the $\mathrm{N}-\mathrm{H}$ vibration of $\mathrm{NH}_{2}$ groups [27] and a peak at $1315 \mathrm{~cm}^{-1}$ related to C-S bond [28]. Peaks at 1629 and $1040 \mathrm{~cm}^{-1}$ are ascribed to -OH of water molecules [29] and C-N vibration [30]. Also, peaks at 2850, and $2930 \mathrm{~cm}^{-1}$ are related to the methyl-stretching bonds [31] and the peak at 3400 $\mathrm{cm}^{-1}$ is ascribed to the O-H stretching of water molecules absorbed into sample [32]. In Au-G4N, Michael addition reaction of $\mathrm{ACN}$ and $-\mathrm{NH}_{2}$ groups results in disappearance of characteristic peak of amine groups at $1465 \mathrm{~cm}^{-1}$ and appearance of a new peak at $2220 \mathrm{~cm}^{-1}$ related to nitrile groups of half-generation PPI dendrimer [33]. After reduction of nitrile groups to primary amines, peak at $2220 \mathrm{~cm}^{-1}$ disappears and peaks at 3260,3130 and $1454 \mathrm{~cm}^{-1}$ related to primary amines appears again. Conjugation of FA onto the surface of nanoparticles leads to appearance of peaks at 1690 and $1626 \mathrm{~cm}^{-1}$ related to amide bonding within FA structure [34] and peaks at 3050 and $1576 \mathrm{~cm}^{-}$ ${ }^{1}$ due to aromatic rings of FA structure [35]. Also, peak at $3330 \mathrm{~cm}^{-1}$ is ascribed to the amine groups within the structure of FA [36].

\section{Figure 4: FT-IR spectra of different modified AuNPs}

TGA were also performed on the modified Au nanoparticles to prove the success of grafting process as shown in Figure 5-A. Also, TGA results are summarized in Table 1. According to the results, Au-NH 2 nanoparticles showed 18.50 wt. $\%$ mass loss $\left(120-700{ }^{\circ} \mathrm{C}\right)$ whereas mass loss of dendrimer-grafted AuNPs increased exponentially with increasing dendrimer generation as depicted in Figure 5-B $\left(\mathrm{y}=23.1442 \mathrm{e}^{0.4066 \mathrm{x}}\right.$, where $\mathrm{y}$ is the weight losses $(\%)$ and $\mathrm{x}$ is the number 
of the generation, $\mathrm{R}^{2}=0.9118$ ). This is the best proof of successful grafting of PPI dendrimers onto the surface of AuNPs [37-38]. Moreover, grafting density (GD) of grafted PPI dendrimers was calculated based on Equation (5) in each modification step [39]:

$G D(\mathrm{~mol} / \mathrm{g}$ of $\mathrm{Au})=\frac{\left(\frac{W_{i}-W_{i-1}}{100-W_{i}}\right)}{M}$

where, $W_{i}, W_{i-1}$ and $M$ are mass losses $\left(120-700{ }^{\circ} \mathrm{C}\right)$ and molecular weight of degradable moiety added in each modification step respectively. According to the results, GD of cysteamine groups was calculated $3.0 \mathrm{mmol} / \mathrm{g}$ of Au and grafting density increased exponentially to $48.2 \mathrm{mmol} / \mathrm{g}$ of Au for Au-G4A nanoparticles (Table 1). When GD is plotting versus generation, an exponential increment of GD versus generation progression is observed $\left(y=2.9719 \mathrm{e}^{0.6949 x}\right.$, where $\mathrm{y}$ is the GD $(\mathrm{mmol} / \mathrm{g}$ of $\mathrm{Au})$ and $\mathrm{x}$ is the number of the generation, $\left.\mathrm{R}^{2}=0.9999\right)$. In this case, exponent of the equation is 0.6949 that is so close to the ideal exponent (0.6931). Conclusively, dendrimer growth from the surface of AuNPs has been occurred ideally and synthesis has been progressed successfully.

Figure 5: A) TGA thermograms of Au-NH2, Au-G1A, Au-G2A, Au-G3A, and Au-G4A; B) Weight loss and GD versus generation progression for modified AuNPs 
Table 1: Summarized TGA results of modified AuNPs

\begin{tabular}{|l|c|c|}
\hline Sample & $\mathbf{W}_{\mathbf{i}}$ (wt. \%) & GD (mmol/g of Au) \\
\hline Au-NH2 & 18.50 & 3.0 \\
\hline Au-G1A & 39.52 & 5.9 \\
\hline Au-G2A & 64.58 & 12.0 \\
\hline Au-G3A & 85.26 & 23.7 \\
\hline Au-G4A & 96.17 & 48.2 \\
\hline
\end{tabular}

\subsection{Drug loading}

Drug loading content (DL) of Au-G4A and Au-G4F were calculated by Equations (6) [40]:

$D L(m g / m g)=\frac{\text { Weight of the drug in nanoparticles }}{\text { Weight of the nanoparticles }}$

DL was calculated $0.033 \pm 0.03$ and $0.045 \pm 0.02$ for Au-G4A and Au-G4F respectively. The amount of drug loading was significantly higher (36.4\%) for Au-G4F compared with Au-G4A. This is originated from better interaction of drug with surface terminal groups [6] due to folate conjugation onto the dendrimer surface. Also, noncovalent interactions such as hydrogen bonding and hydrophobic interaction between DOX and PPI dendrimers lead to physical binding of drug molecules inside the core and surface-engineered groups of dendrimer. Moreover, folate conjugation on the dendrimer surface prevents leaving the DOX molecules from system due to steric hindrance [19]. 


\subsection{In vitro drug-release studies}

To study the feasibility of synthesized hybrid nanoparticles as anti-cancer drug carriers, the in vitro release behavior under a simulated physiological condition $(\mathrm{pH}=7.4)$ and an acidic environment $(\mathrm{pH}=5.3)$ was investigated at $37^{\circ} \mathrm{C}$ (Figure 6). The results showed no significant burst release for $\mathrm{Au}-\mathrm{G} 4 \mathrm{~A}$ and $\mathrm{Au}-\mathrm{G} 4 \mathrm{~F}$ in each condition. This can be ascribed to the loading of the DOX in the cavities of the dendrimers which act as sink to retain the drug molecules for a long duration. However, free drug showed monophasic rapid release $(97.2 \%$ in $4 \mathrm{~h}$ at $\mathrm{pH}=5.3$ and $83.1 \%$ in $7 \mathrm{~h}$ at $\mathrm{pH}=7.4$ ). As a result, synthesized hybrid nanoparticles act as drug-retentive nanocarriers for DOX. Also, Au-G4A showed higher drug release percentage compared to FA-conjugated nanocarriers (Au-G4F) at different $\mathrm{pH}$ values. This is because of steric hindrance on the dendrimer surface originated from FA conjugation that acts as a barrier to prevent leaving the DOX molecules from system. However, the DOX release was more rapid for both conjugated and nonconjugated nanostructures at lower $\mathrm{pH}$ value. The possible reason of this phenomenon is dissolution of DOX water at low $\mathrm{pH}[41]$ whereas DOX becomes positively charged and more soluble in and diffuses more easily from the dendrimer structure to aqueous media. Also, at low $\mathrm{pH}$ values, dendrimer cavities become positively charged due to existence of tertiary amines.

\section{Figure 6: The DOX release behavior of synthesized hybrid nanoparticles}

\subsection{Drug release kinetics}

Mathematical models are effective tools to investigate the release kinetics of drugs from nanocarriers. Among different models, zero-order, first-order, Higuchi, Korsmeyer-Peppas and Hixson-Crowell models are the most well-known models used in variety of systems [42-44]. In 
this work, the release kinetics has been investigated using these models. The release kinetics and parameters of each model have been obtained by linear regression analysis and the coefficients of correlation $\left(\mathrm{R}^{2}\right)$ have been used to verify the accuracy of the fittings, as shown in Table 2 and Figures S1-S5. According to the regression results, the zero-order, first-order and Hixson-Crowell models are not appropriate models to fit release data through hybrid gold/dendrimer nanostructures due to the source of these models. The zero-order model is useful in slow release kinetics. However, gold/dendrimer hybrid nanoparticles show rapid drug release due to a rapid change in solubility in aqueous media especially at low $\mathrm{pH}$ values. Also, the first-order model is applied in porous matrices which are not soluble in release media. Additionally, Hixson-Crowell model can be applied when the surface area and diameter of the drug matrix change with time. None of these three models are the case of study in our work and also, our data show no proper fitting via these systems. However, free drug release systems and drug release from dendritic structures can be fitted by Higuchi and Korsmeyer-Peppas models at both $\mathrm{pH}$ values. Korsmeyer-Peppas model is applicable for drug release from a polymeric matrix. Although no polymeric matrix presents in free drug release system, dialysis bag may behave as polymeric matrix in these systems. Also, Higuchi model describes drug dissolution from matrix into release media. Herein, the most important reason for DOX release from the cavities of dendrimers is the better solubility of DOX in release media than main structure of dendrimers. Conclusively, Korsmeyer-Peppas and Higuchi models describes the DOX release behavior from conjugated and nonconjugated samples at different $\mathrm{pH}$ values. 
Table 2: Mathematical models' correlation coefficients $\left(\mathbf{R}^{2}\right)$ and release exponents for Au-

G4A and Au-G4F hybrid nanoparticles

\begin{tabular}{|c|c|c|c|c|c|c|c|c|}
\hline \multirow{3}{*}{$\begin{array}{c}\text { Kinetics } \\
\text { model }\end{array}$} & \multirow[t]{3}{*}{ Equation [33-35] } & \multirow[t]{3}{*}{ Parameter } & \multicolumn{6}{|c|}{ Value } \\
\hline & & & \multicolumn{3}{|c|}{$\mathrm{pH}=5.3$} & \multicolumn{3}{|c|}{$\mathrm{pH}=7.4$} \\
\hline & & & $\begin{array}{l}\text { Free } \\
\text { drug }\end{array}$ & $\begin{array}{l}\text { Au- } \\
\text { G4A }\end{array}$ & $\begin{array}{l}\text { Au- } \\
\text { G4F }\end{array}$ & $\begin{array}{l}\text { Free } \\
\text { drug }\end{array}$ & $\begin{array}{l}\text { Au- } \\
\text { G4A }\end{array}$ & $\begin{array}{l}\text { Au- } \\
\text { G4F }\end{array}$ \\
\hline \multirow[t]{2}{*}{ Zero-order } & \multirow[t]{2}{*}{$Q_{t}=Q_{0}+K_{0} t^{*}$} & $R^{2}$ & 0.9530 & 0.901 & 0.901 & 0.943 & 0.912 & 0.898 \\
\hline & & $K_{0}$ & 22.924 & 1.090 & 0.944 & 9.626 & 0.669 & 0.500 \\
\hline \multirow[t]{2}{*}{ First-order } & \multirow{2}{*}{$\log C=\log C_{0}+\frac{K}{2.203} t$} & $R^{2}$ & 0.807 & 0.749 & 0.758 & 0.821 & 0.785 & 0.748 \\
\hline & & $K$ & 0.463 & 0.033 & 0.037 & 0.198 & 0.033 & 0.033 \\
\hline \multirow[t]{2}{*}{ Higuchi } & \multirow[t]{2}{*}{$f_{t}=K_{H} t^{1 / 2 * * *}$} & $R^{2}$ & 0.991 & 0.975 & 0.973 & 0.994 & 0.979 & 0.973 \\
\hline & & $K_{H}$ & 60.960 & 9.667 & 8.364 & 32.069 & 5.909 & 4.441 \\
\hline \multirow[t]{3}{*}{ Korsmeyer- } & \multirow{3}{*}{$\frac{M_{t}}{M_{\infty}}=a t^{n \dagger}$} & $R^{2}$ & 0.977 & 0.970 & 0.974 & 0.994 & 0.983 & 0.970 \\
\hline & & $a M_{\infty}$ & 36.224 & 6.486 & 4.539 & 3.334 & 4.121 & 3.289 \\
\hline & & $n$ & 0.805 & 0.612 & 0.660 & 0.523 & 0.597 & 0.590 \\
\hline \multirow{2}{*}{$\begin{array}{l}\text { Hixson- } \\
\text { Crowell }\end{array}$} & \multirow[t]{2}{*}{$Q_{t}^{1 / 3}=Q_{0}^{1 / 3}+k t^{t}$} & $R^{2}$ & 0.870 & 0.812 & 0.819 & 0.869 & 0.837 & 0.809 \\
\hline & & $k$ & 0.567 & 0.036 & 0.036 & 0.244 & 0.030 & 0.027 \\
\hline
\end{tabular}

${ }^{*} \mathrm{~K}_{0}$ is the release kinetic constant.

${ }^{* *} \mathrm{~K}$ is the release kinetic constant.

*** $\mathrm{K}_{\mathrm{H}}$ is the Higuchi's release kinetic constant.

${ }^{\dagger} \mathrm{M}_{\mathrm{t}} / \mathrm{M}_{\infty}$ is a fraction of drug released at time $\mathrm{t}$, $\mathrm{a}$ is the release rate constant and $\mathrm{n}$ is the release exponent.

$\$ \mathrm{k}$ is the release kinetic constant. 


\section{Conclusions}

AuNPs with diameter about $15 \mathrm{~nm}$ were synthesized and modified with $4^{\text {th }}$-generation PPI dendrimers. Then, peripheral amine groups were conjugated with folic acid and the effect of bioconjugation on drug release behavior was investigated. UV-vis. spectra, AFM, TEM, FT-IR and TGA results proved the successful synthesis of hybrid nanoparticles. DOX-loaded Au-G4A and $\mathrm{Au}-\mathrm{G} 4 \mathrm{~F}$ samples were exposed to $\mathrm{pH}$ variations to investigate the drug release behavior of dendrimer-grafted Au nanoparticles. Drug loading content was found 0.033 and 0.045 (mg of drug/mg of dendrimers) for Au-G4A and Au-G4F nanoparticles respectively. As a result, the amount of drug loading was significantly higher for Au-G4F nanoparticles with respect to AuG4A nanoparticles due to FA conjugation onto the dendrons' surface. Also, at different pH values, nonconjugated structures show higher cumulative drug release with respect to FA-conjugated systems because of steric hindrance on the dendrimer surface due to FA conjugation that prevents the DOX molecules from leaving the nanoconjugate system. Additionally, by decreasing the $\mathrm{pH}$ value of release media, the DOX release was more rapid in conjugated and nonconjugated nanostructures. Also, the release kinetics were studied via different models. As a result, both Higuchi and Korsmeyer-Peppas models were fitted on data to describe the release kinetics of DOX from hybrid gold/dendritic nanostructures.

Funding: This work was supported by the Iran National Science Foundation (INSF) [grant number 95833440]. 


\section{References}

[1] L. Dykman, N. Khlebtsov, Gold nanoparticles in biomedical applications: recent advances and perspectives, Chem. Soc. Rev. 41 (2012) 2256-2282. DOI: 10.1039/C1CS15166E

[2] M. Mohammadi, M. Salami-Kalajahi, R. Roghani-Mamaqani, M. Golshan, Synthesis and investigation of dual $\mathrm{pH}$ - and temperature-responsive behaviour of poly[2-(dimethylamino)ethyl methacrylate]-grafted gold nanoparticles, Appl. Organomet. Chem. 2017, DOI: 10.1002/aoc.3702.

[3] P. Zijlstra, P. M. R. Paulo, M. Orrit, Optical detection of single non-absorbing molecules using the surface plasmon resonance of a gold nanorod, Nat. Nanotechnol. 7 (2012) 379-382. DOI: 10.1038/nnano.2012.51.

[4] A. M. Alkilany, L. B. Thompson, S. P. Boulos, P. N. Sisco, C. J. Murphy, Gold nanorods: Their potential for photothermal therapeutics and drug delivery, tempered by the complexity of their biological interactions, Adv. Drug Deliv. Rev. 64 (2012) 190-199. DOI: 10.1016/j.addr.2011.03.005.

[5] C. Jinping, G. Yan-Juan, C. S. Han, W. Wing-Tak, Surface Functionalized Gold Nanoparticles for Drug Delivery, J. Biomed. Nanotechnol. 9 (2013) 1362-1369. DOI: 10.1166/jbn.2013.1536.

[6] P. Kesharwani, K. Jain, N. K. Jain, Dendrimer as nanocarrier for drug delivery, Prog. Polym. Sci. 39 (2014) 268-307. DOI: 10.1016/j.progpolymsci.2013.07.005.

[7] J. Zhu, X. Shi, Dendrimer-based nanodevices for targeted drug delivery applications, J. Mater. Chem. B 1 (2013) 4199-4211. DOI: 10.1039/C3TB20724B.

[8] F. Samadaei, M. Salami-Kalajahi, H. Roghani-Mamaqani, Grafting of Poly(Acrylic Acid) onto Poly(amidoamine)-Functionalized Graphene Oxide via Surface-mediated Reversible Addition- 
Fragmentation Chain Transfer Polymerization, Int. J. Polym. Mater. 65 (2016) 302-309. DOI: 10.1080/00914037.2015.1119686.

[9] M. Golshan, M. Salami-Kalajahi, R. Roghani-Mamaqani, M. Mohammadi, Synthesis of poly(propylene imine) dendrimer via homogeneous reduction process by lithium aluminum hydride: bioconjugation with folic acid and DOX release kinetics, Appl. Organomet. Chem. 2017, DOI: 10.1002/aoc.3789.

[10] P. Kesharwani, V. Mishra, N. K. Jain, Generation dependent hemolytic profile of folate engineered poly(propyleneimine) dendrimer, J. Drug Deliv. Sci. Tech. 28 (2015) 1-6. DOI: 10.1016/j.jddst.2015.04.006.

[11] P. Kesharwani, R. K. Tekade, N. K. Jain, Generation Dependent Safety and Efficacy of Folic Acid Conjugated Dendrimer Based Anticancer Drug Formulations, Pharm. Res. 32 (2015) 14381450. DOI: $10.1007 / \mathrm{s} 11095-014-1549-2$.

[12] M. Banaei, M. Salami-Kalajahi, Synthesis of poly(2-hydroxyethyl methacrylate)-grafted poly(aminoamide) dendrimers as polymeric nanostructures, Colloid Polym. Sci., 2015, 293, 15531559. DOI: $10.1007 / \mathrm{s} 00396-015-3559-\mathrm{y}$.

[13] M. Torkpur-Biglarianzadeh, M. Salami-Kalajahi, Multilayer Fluorescent Magnetic Nanoparticles with Dual Thermoresponsive and pH-sensitive Polymeric Nanolayers as Anticancer Drug Carriers, RSC Adv. 5 (2015) 29653-29662. DOI: 10.1039/C5RA01444A.

[14] M. Nemanashi, R. Meijboom, Synthesis and characterization of $\mathrm{Cu}, \mathrm{Ag}$ and Au dendrimerencapsulated nanoparticles and their application in the reduction of 4-nitrophenol to 4aminophenol, J. Colloid Interface Sci. 389 (2013) 260-267. DOI: 10.1016/j.jcis.2012.09.012. 
[15] T. Ndlovu, O. A. Arotiba, Poly(propylene imine) Dendrimer and Gold Nanoparticles as Antipassivating Electrode Modifiers in Phenol Sensing - a Case Study of 4-Chorophenol, Int. J. Electrochem. Sci. 10 (2015) 8224-8235.

[16] L. Kong, Y. Wu, C. S Alves, X. Shi, Efficient delivery of therapeutic siRNA into glioblastoma cells using multifunctional dendrimer-entrapped gold nanoparticles, Nanomedicine (2016) DOI: 10.2217/nnm-2016-0240.

[17] M. Banaei, M. Salami-Kalajahi, A "Grafting to" Approach to Synthesize Low Cytotoxic Poly(aminoamide)-Dendrimer-grafted $\mathrm{Fe}_{3} \mathrm{O}_{4}$ Magnetic Nanoparticles, Adv. Polym. Technol., 2016, DOI: 10.1002/adv.21741.

[18] J. Turkevich, P. C. Stevenson, J. Hillier, A study of the nucleation and growth processes in the synthesis of colloidal gold, Discuss. Faraday Soc. 11 (1951) 55-75. DOI: 10.1039/DF9511100055.

[19] A. Agarwal, U. Gupta, A. Asthana, N. K. Jain, Dextran conjugated dendritic nanoconstructs as potential vectors for anti-cancer agent, Biomaterials 30 (2009) 3588-3596. DOI: 10.1016/j.biomaterials.2009.03.016.

[20] X. Shi, W. Lesniak, M. T. Islam, M. C. MuÑiz, L. P. Balogh, J. R. Baker Jr., Comprehensive characterization of surface-functionalized poly(amidoamine) dendrimers with acetamide, hydroxyl, and carboxyl groups, Colloids Surf. A Physicochem. Eng. Asp. 272 (2006) 139-150. DOI: 10.1016/j.colsurfa.2005.07.031.

[21] Y. Zhang, T. P. Thomas, A. Desai, H. Zong, P. R. Leroueil, I. J. Majoros, J. R. Baker Jr., Targeted Dendrimeric Anticancer Prodrug: A Methotrexate-Folic Acid-Poly(amidoamine) Conjugate and a Novel, Rapid, "One pot” Synthetic Approach, Bioconjug. Chem. 21 (2010) 489495. DOI: 10.1021/bc9003958. 
[22] Y. Gao, Z. Li, X. Xie, C. Wang, J. You, F. Mo, B. Jin, J. Chen, J. Shao, H. Chen, L. Jia, Dendrimeric anticancer prodrugs for targeted delivery of ursolic acid to folate receptor-expressing cancer cells: Synthesis and biological evaluation, Eur. J. Pharm. Sci. 70 (2015) 55-63. DOI: 10.1016/j.ejps.2015.01.007.

[23] P. Du, H. Li, Z. Mei, S. Liu, Electrochemical DNA biosensor for the detection of DNA hybridization with the amplification of $\mathrm{Au}$ nanoparticles and $\mathrm{CdS}$ nanoparticles, Bioelectrochemistry 75 (2009) 37-43. DOI: 10.1016/j.bioelechem.2009.01.003.

[24] D. Ghosh, N. Chattopadhyay, Gold Nanoparticles: Acceptors for Efficient Energy Transfer from the Photoexcited Fluorophores, Opt. Photonics J. 3 (2013) 18-26. DOI: 10.4236/opj.2013.31004.

[25] A. Sharma, Z. Matharu, G. Sumana, P. R. Solanki, C. G. Kim, B. D. Malhotra, Antibody immobilized cysteamine functionalized-gold nanoparticles for aflatoxin detection, Thin Solid Films 519 (2010) 1213-1218. DOI: 10.1016/j.tsf.2010.08.071.

[26] M. Safajou-Jahankhanemlou, F. Abbasi, M. Salami-Kalajahi, Synthesis and characterization of thermally expandable PMMA-based microcapsules with different cross-linking density, Colloid Polym. Sci. 294 (2016) 1055-1064. DOI: 10.1007/s00396-016-3862-2.

[27] M. Yu, C. Zhou, J. Liu, J. D. Hankins, J. Zheng, Luminescent Gold Nanoparticles with pHDependent Membrane Adsorption, J. Am. Chem. Soc. 133 (2011) 11014-11017. DOI: 10.1021/ja201930p.

[28] J. U. Lee, W. Lee, S. S. Yoon, J. Kim, J. H. Byun, Site-selective immobilization of gold nanoparticles on graphene sheets and its electrochemical properties, Appl. Surf. Sci. 315 (2014) 73-80. DOI: 10.1016/j.apsusc.2014.07.099. 
[29] Y.-M. Wang, Y. Cui, Z.-Q. Cheng, L.-S. Song, Z.-Y. Wang, B.-H. Han, J.-S. Zhu, Poly(acrylic acid) brushes pattern as a 3D functional biosensor surface for microchips, Appl. Surf. Sci. 266 (2013) 313-318. DOI: 10.1016/j.apsusc.2012.12.017.

[30] H. Li, X. Ye, X. Guo, Z. Geng, G. Wang, Effects of surface ligands on the uptake and transport of gold nanoparticles in rice and tomato, J. Hazard. Mater. 314 (2016) 188-196. DOI: 10.1016/j.jhazmat.2016.04.043.

[31] M. Sarsabili, M. Parvini, M. Salami-Kalajahi, P. Ganjeh-Anzabi, In situ reversible additionfragmentation chain transfer polymerization of styrene in the presence of mcm-41 nanoparticles: comparing "grafting from" and "grafting through" approaches, Adv. Polym. Technol. 32 (2013) 21372. DOI: 10.1002/adv.21372.

[32] M. Nikdel, M. Salami-Kalajahi, M. S. Hosseini, Dual thermo-and pH-sensitive poly (2hydroxyethyl methacrylate-co-acrylic acid)-grafted graphene oxide, Colloid Polym. Sci. 292 (2014) 2599-2610. DOI: 10.1007/s00396-014-3313-X.

[33] W. Li, Z. Yang, G. Zhang, Z. Fan, Q. Meng, C. Shen, C. Gao, Stiff metal-organic frameworkpolyacrylonitrile hollow fiber composite membranes with high gas permeability, J. Mater. Chem. A 2 (2014) 2110-2118. DOI: 10.1039/C3TA13781C.

[34] C. Lu, L. R. Bhatt, H. Y. Jun, S. H. Park, K. Y. Chai, Carboxyl-polyethylene glycolphosphoric acid: a ligand for highly stabilized iron oxide nanoparticles, J. Mater. Chem. 22 (2012) 19806-19811. DOI: 10.1039/C2JM34327D.

[35] M. Sarsabili, M. Parvini, M. Salami-Kalajahi, A. Asfadeh, Effect of MCM-41 nanoparticles on the kinetics of free radical and RAFT polymerization of styrene, Iran. Polym. J. 22 (2013) 155163. DOI: $10.1007 / \mathrm{s} 13726-012-0114-2$. 
[36] F. Muhammad, M. Guo, Y. Guo, W. Qi, F. Qu, F. Sun, H. Zhao, G. Zhu, Acid degradable $\mathrm{ZnO}$ quantum dots as a platform for targeted delivery of an anticancer drug, J. Mater. Chem. 21 (2011) 13406-13412. DOI: 10.1039/C1JM12119G.

[37] Y. Zhang, Y. Li, P. Zhang, De novo growth of poly(amidoamine) dendrimers on the surface of multi-walled carbon nanotubes, J. Mater. Sci. 49 (2014) 3469-3477. DOI: 10.1007/s10853-0148059-9.

[38] F. Samadaei, M. Salami-Kalajahi, H. Roghani-Mamaqani, M. Banaei, A Structural Study on Ethylenediamine- and Poly(amidoamine)-functionalized Graphene Oxide: Simultaneous Reduction, Functionalization, and Formation of 3D Structure, RSC Adv. 5 (2015) 71835-71843. DOI: 10.1039/C5RA12086A.

[39] M. Salami-Kalajahi, V. Haddadi-Asl, S. Rahimi-Razin, F. Behboodi-Sadabad, M. Najafi, H. Roghani-Mamaqani, A study on the properties of PMMA/silica nanocomposites prepared via RAFT polymerization, J. Polym. Res. 19 (2012) 9793. DOI: 10.1007/s10965-011-9793-1.

[40] H. Xu, Z. Hou, H. Zhang, H. Kong, X. Li, H. Wang, W. Xie, An efficient Trojan delivery of tetrandrine by poly(N-vinylpyrrolidone)-block-poly( $\varepsilon$-caprolactone) (PVP-b-PCL) nanoparticles shows enhanced apoptotic induction of lung cancer cells and inhibition of its migration and invasion, Int. J. Nanomedicine 9 (2014) 231-242. DOI: 10.2147/IJN.S55541.

[41] L.-B. Chen, F. Zhang, C.-C. Wang, Rational Synthesis of Magnetic Thermosensitive Microcontainers as Targeting Drug Carriers, Small 5 (2009) 621-628. DOI: 10.1002/smll.200801154.

[42] H. R. Aucoin, A. N. Wilson, A. M. Wilson, K. Ishihara, A. Guiseppi-Elie, Release of Potassium Ion and Calcium Ion from Phosphorylcholine Group Bearing Hydrogels, Polymers 5 (2013) 1241-1257. DOI: 10.3390/polym5041241. 
[43] Y. Fu, W. J. Kao, Drug Release Kinetics and Transport Mechanisms of Non-degradable and Degradable Polymeric Delivery Systems, Expert Opin. Drug Deliv. 7 (2010) 429-444. DOI: $10.1517 / 17425241003602259$.

[44] L. Tao, J. W. Chan, K. E Uhrich, Drug loading and release kinetics in polymeric micelles: Comparing dynamic versus unimolecular sugar-based micelles for controlled release, J. Bioact. Compat. Polym. 31 (2016) 227-241. DOI: 10.1177/0883911515609814. 


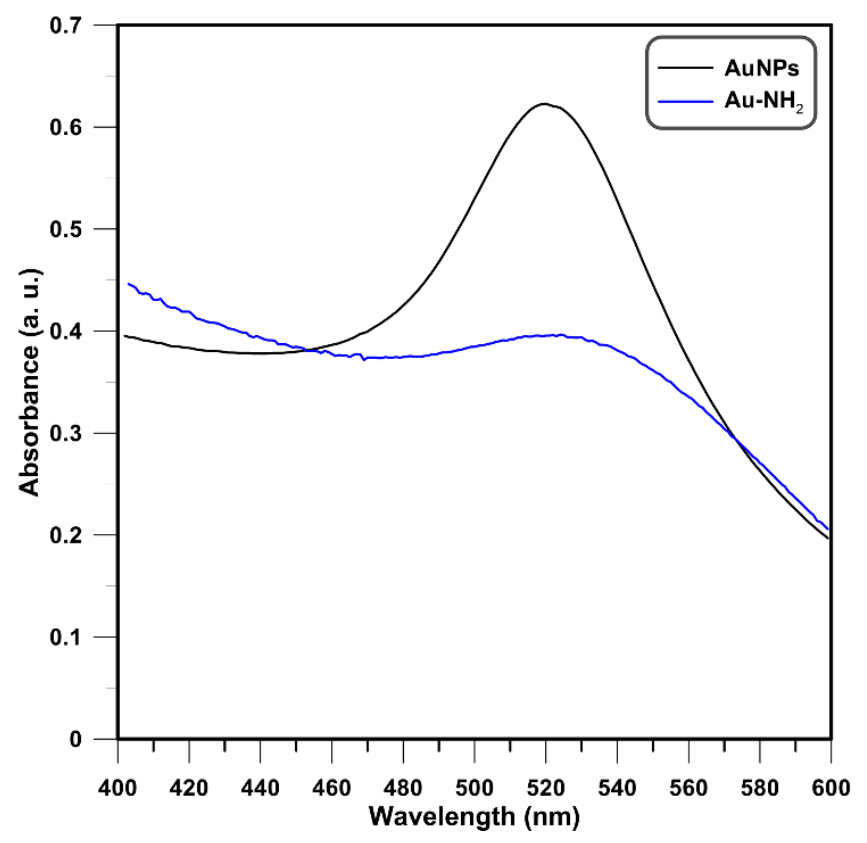



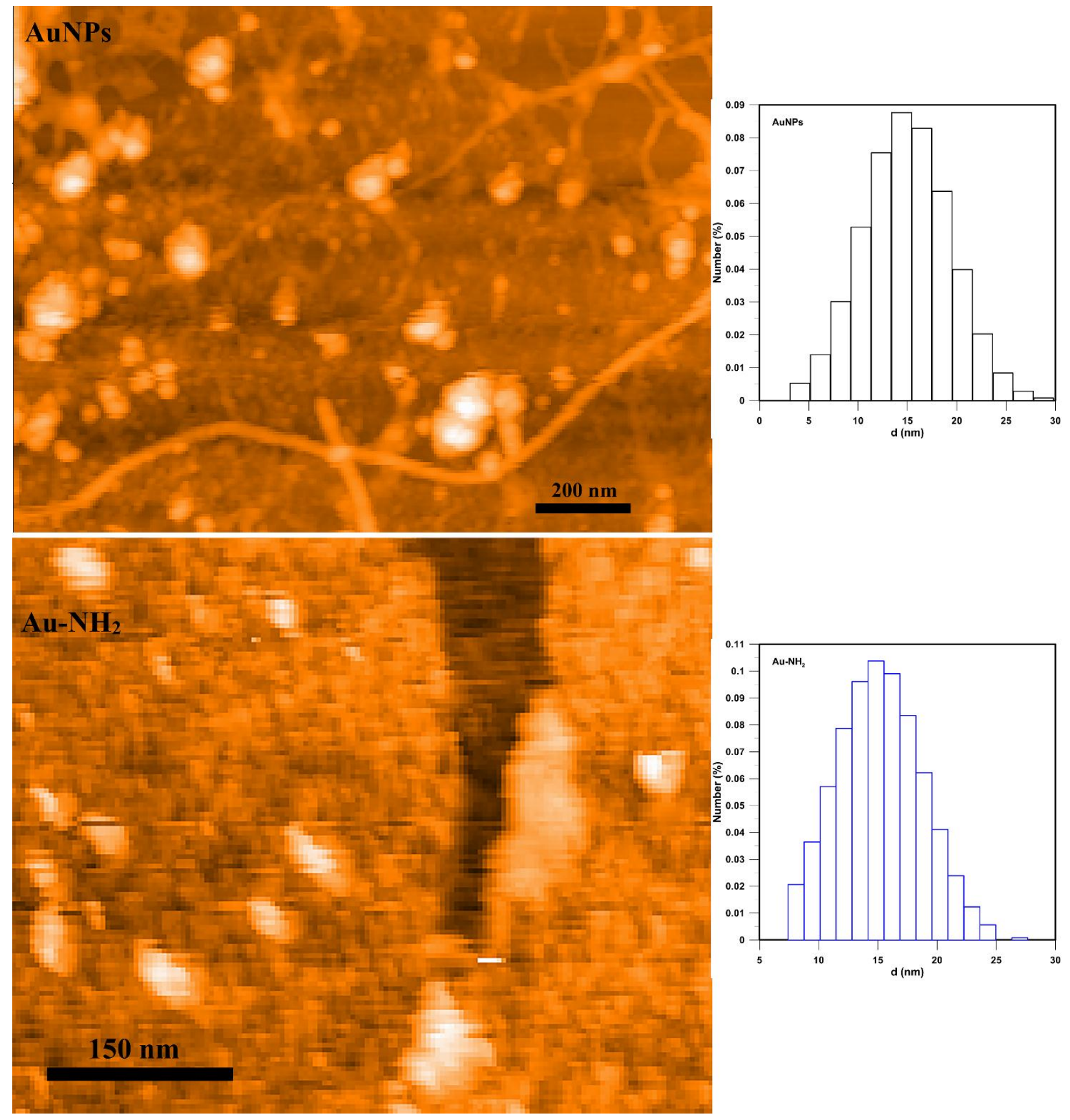


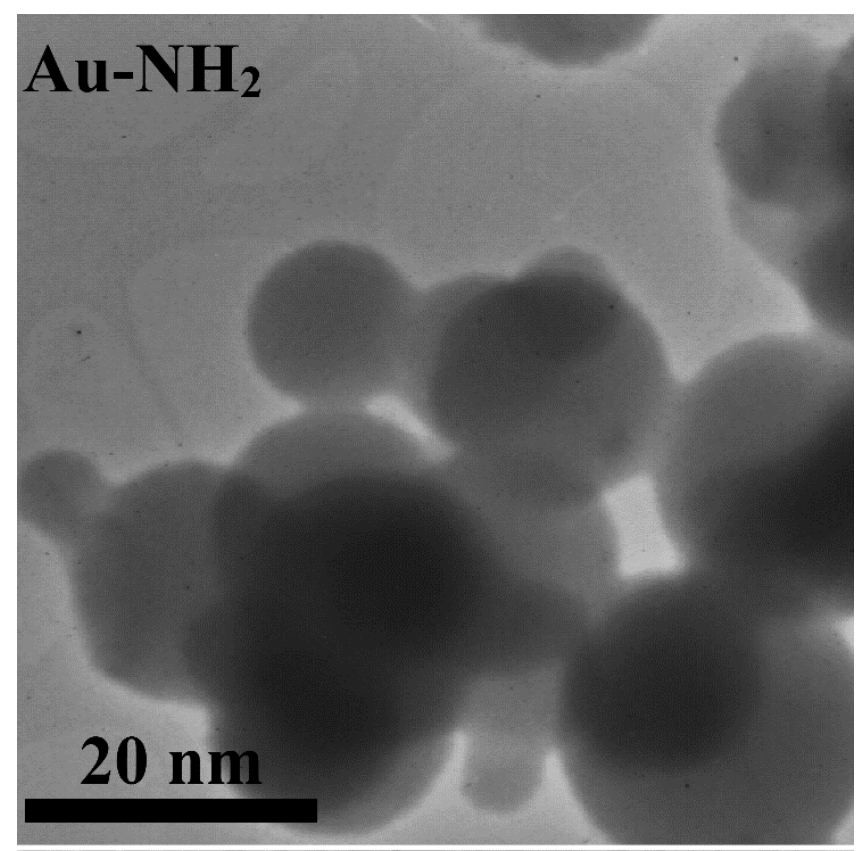

Au-G4A

$10 \mathrm{~nm}$ 


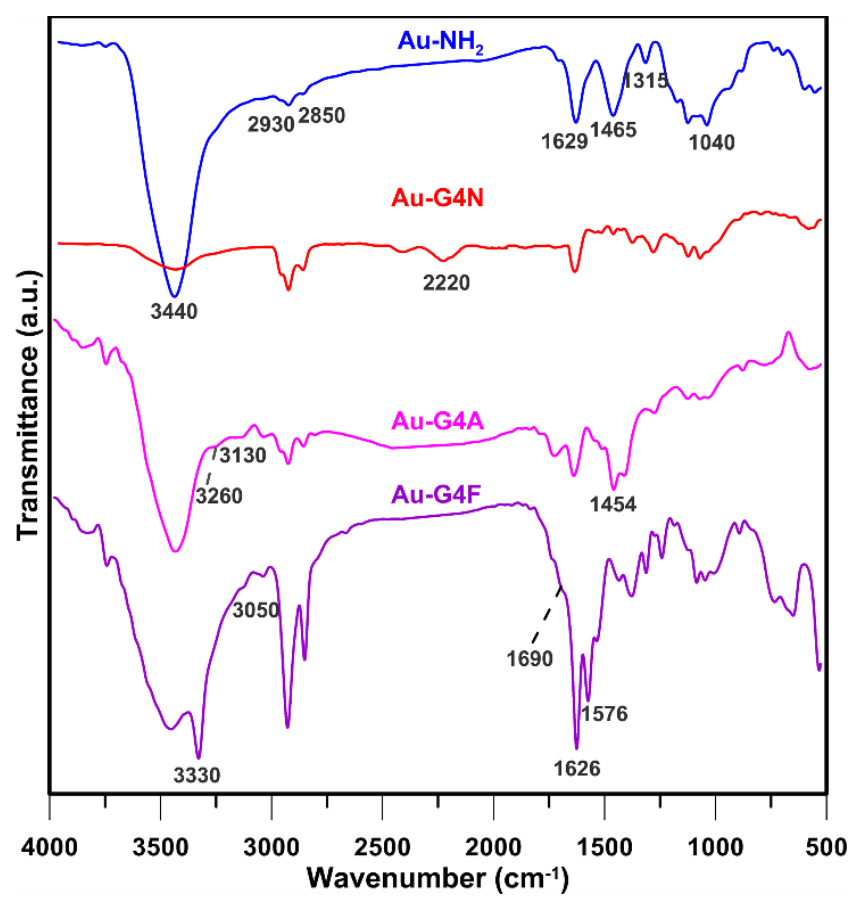



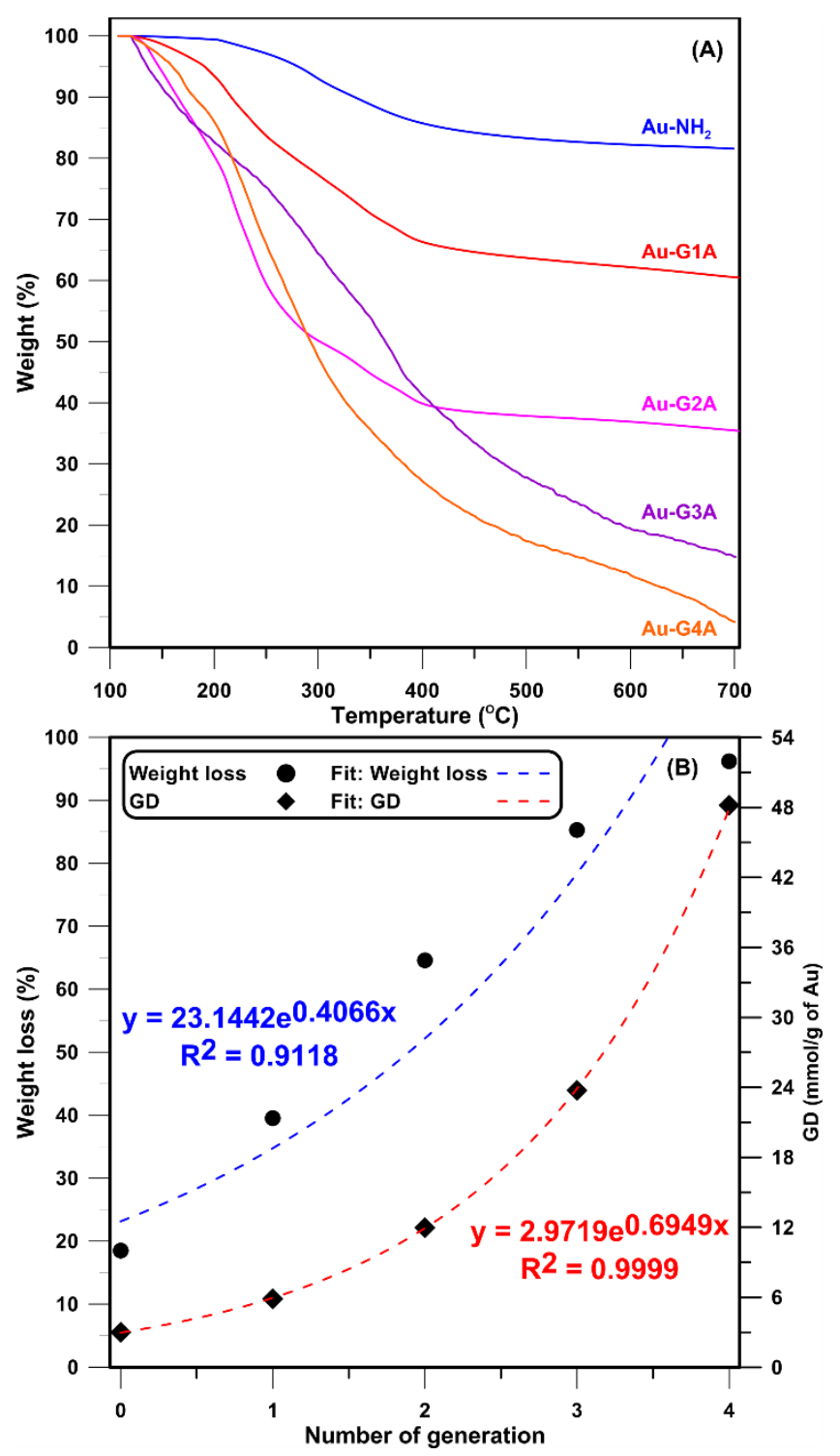


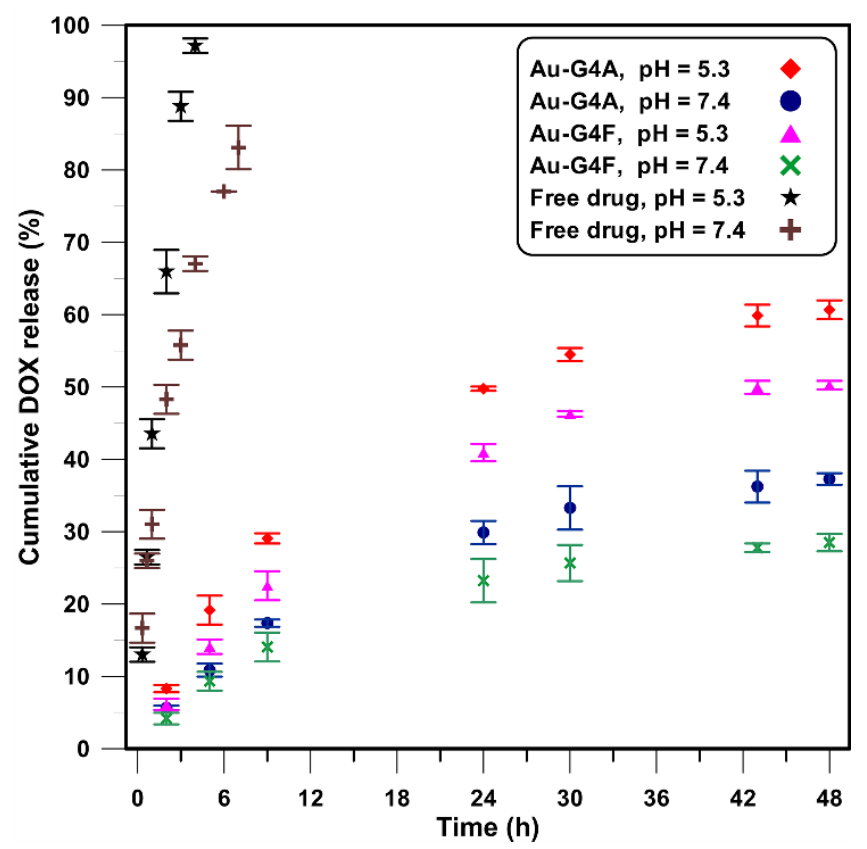




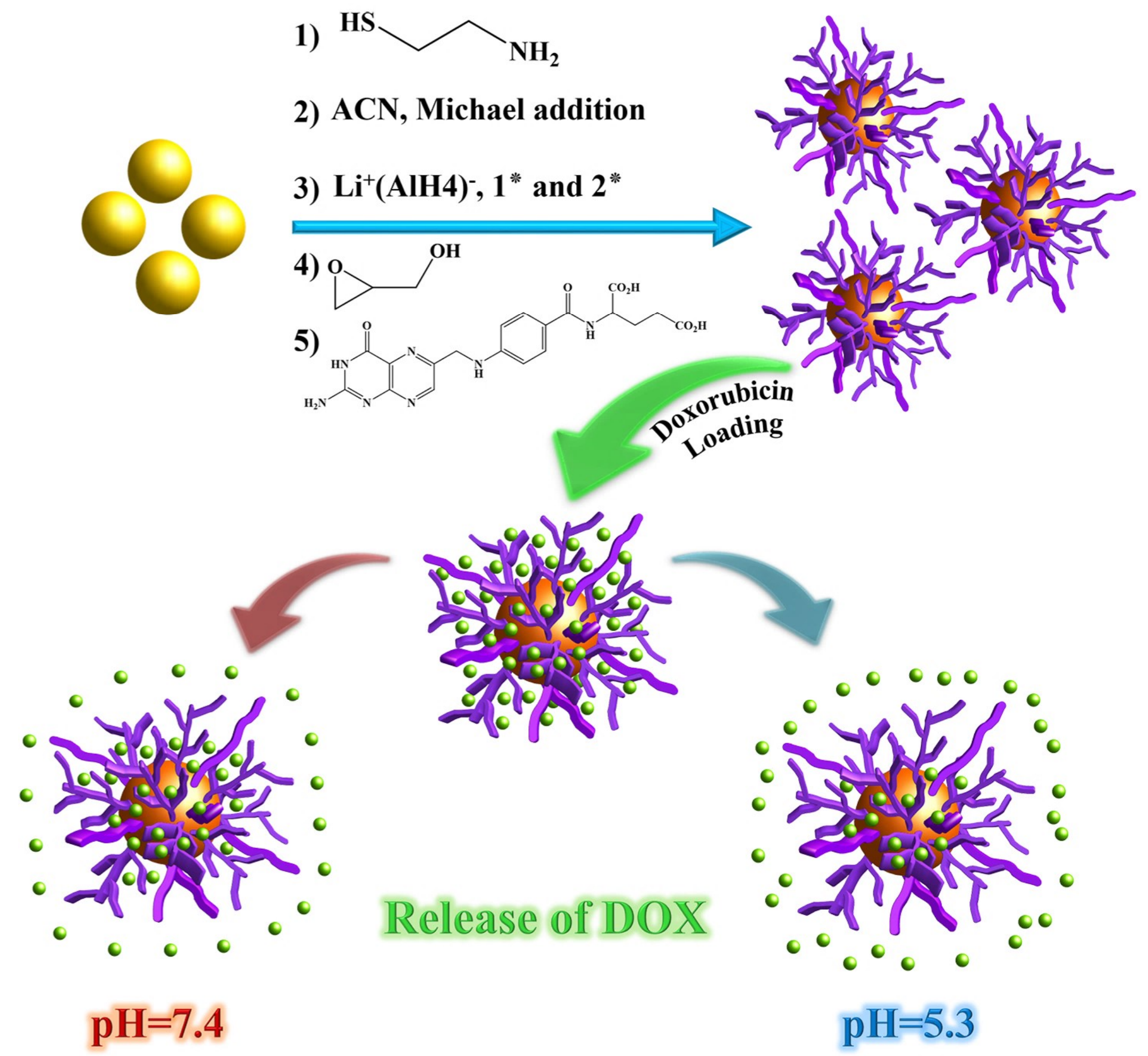

